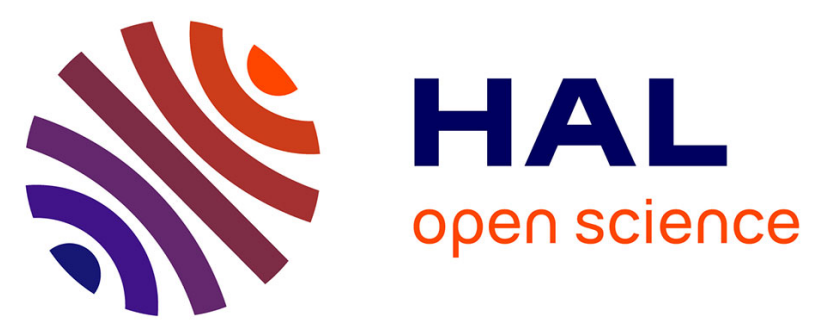

\title{
Increased intracellular survival of Salmonella Typhimurium ST313 in HIV-1-infected primary human macrophages is not associated with Salmonella hijacking the HIV compartment
}

G. Lê-bury, C. Deschamps, C. Kizilyaprak, W. Blanchard, J. Daraspe, Audrey Dumas, M.A. Gordon, J.C.D. Hinton, B.M. Humbel, F. Niedergang

\section{To cite this version:}

G. Lê-bury, C. Deschamps, C. Kizilyaprak, W. Blanchard, J. Daraspe, et al.. Increased intracellular survival of Salmonella Typhimurium ST313 in HIV-1-infected primary human macrophages is not associated with Salmonella hijacking the HIV compartment. Biology of the Cell, 2020, 112 (3), pp.92101. 10.1111/boc.201900055 . hal-03093027

\section{HAL Id: hal-03093027 https://hal.science/hal-03093027}

Submitted on 7 Jan 2021

HAL is a multi-disciplinary open access archive for the deposit and dissemination of scientific research documents, whether they are published or not. The documents may come from teaching and research institutions in France or abroad, or from public or private research centers.
L'archive ouverte pluridisciplinaire HAL, est destinée au dépôt et à la diffusion de documents scientifiques de niveau recherche, publiés ou non, émanant des établissements d'enseignement et de recherche français ou étrangers, des laboratoires publics ou privés. 
Title

Increased intracellular survival of Salmonella Typhimurium ST313 in HIV-1infected primary human macrophages is not associated with Salmonella hijacking the HIV compartment

Running Title (50 characters)

Intra-macrophage HIV- and Salmonella-compartments

\section{Authors}

G. Lê-Bury ${ }^{1 \#}$, C. Deschamps ${ }^{1 \#}$, C. Kizilyaprak², W. Blanchard ${ }^{2}$, J. Daraspe ${ }^{2}$, A. Dumas $^{1}$, J. C. D. Hinton ${ }^{3}$, M.A. Gordon ${ }^{3}$, B. M. Humbel ${ }^{2,4}$ and F. Niedergang ${ }^{1 *}$.

1. Université de Paris, Institut Cochin, INSERM, U1016, CNRS, UMR 8104, F-75014 Paris, France.

2. Université de Lausanne, Faculté de Biologie et de Médecine, Electron Microscopy Facility.

3. Institute of Integrative Biology, University of Liverpool, United Kingdom.

4. Institute of Infection and Global Health, University of Liverpool, United Kingdom.

4. IMG, Okinawa Institute of Science and Technology, Onna-son, Okinawa, Japan.

\# Both authors contributed equally to this work

* Corresponding author:

Dr. Florence Niedergang 
Institut Cochin, Biology of Phagocytes group, Infection, Immunity and Inflammation Department

22, rue Méchain, 75014 Paris, France.

Phone: 0033140516421

Fax: 0033140516430

Email: florence.niedergang@inserm.fr

\section{Keywords (5)}

Macrophages, Human Immunodeficiency Virus type 1 (HIV-1), invasive Salmonella enterica Typhimurium ST313, FIB-SEM

Text 3235 words 


\section{Abstract}

Non-Typhoidal Salmonella (NTS) causes a severe invasive syndrome (iNTS disease) described in HIV-positive adults. The impact of HIV-1 on Salmonella pathogenesis and the molecular basis for the differences between these bacteria and classical diarrhoeal S. Typhimurium remains unclear. Here we show that iNTS-associated $S$. Typhimurium Sequence Type 313 (ST313) bacteria show greater intracellular survival in primary human macrophages, compared with a 'classical' diarrhoeal $S$. Typhimurium ST19 isolate. The increased intracellular survival phenotype of ST313 is more pronounced in HIV-infected macrophages. We explored the possibility that the bacteria take advantage of the HIV-associated viral-containing compartments created in human macrophages that have low $\mathrm{pH}$. Confocal fluorescence microscopy and Focused Ion Beam-Scanning Electron Microscopy (FIB-SEM) tomography showed that Salmonella did not co-localise extensively with HIV-positive compartments. These results indicate that the bacteria do not directly benefit from the niche created by the virus in HIV-1 infected macrophages, and that they might take advantage from a more globally modified host cell. 


\section{INTRODUCTION}

Macrophages are phagocytic cells that populate all tissues and organs of the body where they play important roles in pathogen clearance, removal of cell debris and matrix degradation. Many of these functions rely upon to their capacity to perform phagocytosis, a mechanism of internalisation of large particulate material that relies on the ligation of surface receptors (Niedergang, 2016; Niedergang and Grinstein, 2018).

As with other cells that express the receptor, CD4, and co-receptors, CXCR4 and CCR5, macrophages can be infected by the human immunodeficiency virus type 1 (HIV-1). Macrophages, however, resist the cytopathic effect of the virus and represent one of the viral reservoirs, even in treated patients (Rodrigues et al., 2017). For instance, urethral macrophages were recently reported to constitute a principal site of replication competent HIV-1 reservoir (Ganor et al., 2019).

We and others have previously demonstrated that HIV-1-infected macrophages have impaired phagocytic capacity against a variety of phagocytic targets, coupled with defective phagolysosome maturation and clearance capacity. These defective phagocytic capacities enable bacteria and other opportunistic pathogens to better survive intracellularly (Biggs et al., 1995; Crowe et al., 1994; Dumas et al., 2015; Kedzierska et al., 2003; Torre et al., 2002). Many intracellular pathogens have been associated with opportunistic infections of HIV-infected hosts, including Mycobacterium tuberculosis, Toxoplasma gondii, Candida albicans, Aspergillus fumigatus, etc. In 1990, non-typhoidal salmonellae (NTS) were identified as HIVassociated pathogens in sub-Saharan African adults (Gilks et al., 1990). Later, invasive non-typhoidal Salmonella disease (iNTS) became recognized as a common and recurrent illness among susceptible African children and HIV-infected adults 
(Gordon et al., 2001), and was responsible for 66,000 deaths in Africa in 2017 (Collaborators, 2019). This systemic disease differs from the self-limiting diarrheal disease that has classically been associated with $S$. Typhimurium in immunocompetent individuals (Gilchrist and MacLennan, 2019).

The S. Typhimurium serovar causes two-thirds of iNTS in sub-Saharan Africa, and phylogenetic analysis has revealed that the bacteria represent a distinct pathovariant that is multidrug resistant and belongs to a specific multi-locus sequence type, ST313 (Canals et al., 2019; Kingsley et al., 2009; Okoro et al., 2012; Van Puyvelde et al., 2019). Recent studies have addressed the abilities of the ST313 bacteria to disseminate systemically, as well as their capacity to survive intracellularly in various experimental models, including macrophages (Carden et al., 2015; Herrero-Fresno et al., 2014; Lacharme-Lora et al., 2019; Ramachandran et al., 2015; Singletary et al., 2016). However, the impact of a HIV-pre-infection on these cells remains to be investigated (Le-Bury and Niedergang, 2018).

The HIV-1 lifecycle in macrophages differs to that in CD4+ T cells and model cell lines, where viral assembly and budding takes place at the plasma membrane. In macrophages, the virus buds and is stored in a compartment called Virus-Containing Compartment (VCC) (Tan and Sattentau, 2013). This compartment has unique properties that distinguishes it from other multivesicular bodies: the VCC lacks lysosomal markers, has a non-acidic $\mathrm{pH}$, and is connected to the plasma membrane (Rodrigues et al., 2017). S. Typhimurium also survives in a specific compartment, the Salmonella-Containing Vacuole (SCV), that resists fusion with lysosomal contents (Holden, 2002; Malik-Kale et al., 2011).

Here, we test the hypothesis that in HIV-1 infected macrophages, intracellular $S$. Typhimurium could take advantage of the neutral HIV-1-associated compartment to 
achieve increased survival. The intracellular fate of both pathogens, HIV-1 and S. Typhimurium, were specifically analysed, using confocal fluorescent microscopy and Focused Ion Beam-Scanning Electron Microscopy (FIB-SEM) (Kizilyaprak et al., 2019; Weiner and Enninga, 2019). These experiments revealed that S. Typhimurium survive inside macrophages without directly hijacking the viral compartment in human macrophages.

\section{RESULTS}

\section{Enhanced survival of ST313 bacteria in HIV-infected human primary macrophages}

To characterize the impact of HIV-1 infection on bacterial survival of invasive $S$. Typhimurium, we used an infection model involving primary human monocytederived macrophages that were pre-infected with HIV-1 for 8 days. In this paper, we refer to the un-infected macrophages as $\mathrm{HIV}-1^{-}$, and to the HIV-1-infected macrophages as $\mathrm{HIV}-1^{+}$. The two types of macrophages were then infected with ST313 D23580, or a representative non-invasive isolate ST19 4/74 (Figure 1A). A gentamicin protection assay was performed and results were expressed as Colony Forming Units per $\mathrm{mL}(\mathrm{CFU} / \mathrm{mL})$ (Figure 1B) or fold-change intracellular survival of ST313 versus ST19 bacteria (Figure 1C). In the experimental conditions used, 10$50 \%$ of macrophages were productively infected with HIV-1, and all cells were coinfected with bacteria (Dumas et al., 2015). Initially, similar levels of ST313 and ST19 were internalized into $\mathrm{HIV}-1^{-}$and $\mathrm{HIV}-1^{+}$primary macrophages (Figure 1B "entry"), as reported previously in primary mouse bone marrow-derived macrophages (BMDM) (Carden et al., 2015), but not seen in murine cell lines such as J774 (Ramachandran 
et al., 2015). At later stages of infection, ST313 invasive bacteria showed a clear trend towards a higher ( 2 to 3 times) level of intracellular survival inside human macrophages, compared with reference ST19 bacteria, as in (Ramachandran et al., 2015). Interestingly, this was observed in both $\mathrm{HIV}-1^{-}$and $\mathrm{HIV}-1^{+}$macrophages (Figure 1B-C, “20-24 h"). In addition, despite the relatively low rate of HIV-infection, at $24 \mathrm{~h}$ post-infection, there was a trend for increased survival of ST313 versus ST19 in HIV $-1^{+}$macrophages ( 3 times more), compared to HIV $-1^{-}$cells (2 times more).

Taken together, these data suggest that the ability of ST313 bacteria to survive better than ST19 bacteria within primary human macrophages is enhanced in cells preinfected with HIV-1.

\section{Salmonella Typhimurium and HIV-1 are located in different compartments in primary human macrophages}

The improved survival of ST313 versus ST19 in HIV-infected macrophages raised the possibility that there was a direct connection between the SCV and the non-acidic VCC. To determine if bacteria can 'hijack' the VCC, the localisation of HIV-1 and the ST19 reference bacteria or the ST313 iNTS bacteria was determined on fixed coinfected primary macrophages that were fluorescently-labelled with antibodies targeting the p24 capsid protein of HIV and the Salmonella O-antigen. Co-localisation of the virus and the two types of Salmonella were determined using Pearson correlation coefficients (PCC) (Figure 2A-C). The resulting PCC values were $<0.3$, indicating a low association between HIV and both Salmonella pathovariants (Figure 2C). The values were above zero, so transient contacts between the SCV and VCC compartments cannot be excluded. 
To search for transient connections between the VCC and the bacteria, we analyzed the dynamic behaviour of the two types of pathogens in living macrophages coinfected with HIV-1 NLR4.3 Gag-iGFP and DsRed-expressing ST313 (SSS18 (pMR98)) (Figure 2E). As expected (Bakowski et al., 2008), after internalisation, the bacteria gradually moved towards the cell centre, close to the VCC. However, the fluorescent signals from bacteria and virus originated from different focal planes (3D representation in Figure 2E). The PCC calculated for three different cells remain low during the $6 \mathrm{~h}$ experiment (PCC <0.2), confirming that the bacterial and viral compartments were not closely associated in the co-infected cells over this time period (Figure 2D).

Three-dimensional ultrastructural analysis of Salmonella Typhimurium- and HIV-1-containing compartments in human primary macrophages using FIB-

\section{SEM}

To visualize the pathogen compartments, an electron microscopy analysis was performed. For this, human macrophages were plated on a gridded dish, co-infected with HIV-1 for 8 days and DsRed-expressing ST313 for 6 h post-invasion of bacteria. After fixation, cells were observed with confocal fluorescence microscopy (Figure 3A) and some were selected for FIB-SEM analysis. The images from one co-infected macrophage revealed the location of the VCC and bacterial compartments (Figure $3 \mathrm{~B}$, original dataset stack can be found on line at https://imagerie.cochin.inserm.fr as described in the Methods). The 3D reconstruction of the image stacks highlights the compartments' contours (Figure $3 \mathrm{C}$ and Supp Figure movie 1 on line). The cell appeared to have two nuclei (in blue). Interestingly, the plasma membrane showed extensive ruffles. One striking feature of the reconstructed $3 D$ view is that the 
compartments containing the bacteria were not tight around the microorganisms but rather show large membrane ruffles and extensions (Figure 3C membranes in purple, and bacteria in red, in ii and iii where the membranes are transparent). These are reminiscent of the SIFs (Salmonella-induced filaments)(Knuff and Finlay, 2017), but extended SCV membranes decorated with late-endocytic markers were not detected in macrophages by fluorescence microscopy (not shown). No direct membrane continuity was observed between the viral compartment, (Figure $3 \mathrm{C}$ green) and the bacterial compartments (Figure $3 \mathrm{C}$ purple), although transient connections between the compartments cannot be excluded. These results demonstrate that the SCV is not extensively connected with the VCC in co-infected macrophages at the time points analysed. We conclude that the enhanced ST313 bacterial survival and replication observed in HIV-1-infected macrophages does not reflect direct hijacking of the VCC viral compartment by Salmonella. 


\section{Discussion}

Here we analysed the intracellular fate of S. Typhimurium ST313 in HIV-1 ${ }^{-}$and HIV$1^{+}$primary human macrophages. We found that S. Typhimurium ST313 D23580 showed a trend of increased survival and replication in human monocyte-derived macrophages, compared with ST19 4/74. This is consistent with a previous report that involved intracellular infection experiments with S. Typhimurium ST313 in the THP-1 human monocytic cell line, in PBMCs from healthy human donors and in murine peritoneal macrophages (Ramachandran et al., 2015). We have now discovered that ST313 shows even higher levels of survival than ST19 in HIVinfected macrophages (Figure 1C "20-24h").

In this study, the temporal and spatial distribution of cellular structures containing two different pathogens, HIV-1 and S. Typhimurium in primary human macrophages, were analysed using both live fluorescence imaging and FIB/SEM. The combination of dynamic fluorescence imaging and the high resolution FIB/SEM approach allowed us to obtain a unique perspective on the two compartments. Although the VCC is located in a perinuclear region (Rodrigues et al., 2017), close to where the bacterial compartment traffics after bacterial invasion of the macrophage, the two fluorescence signals did not show significant co-localisation (Figure 2).

Fluorescence microscopy, however, was limited by the ability to detect the fluorescently-labelled microorganisms, namely the GFP-labelled viral capsids and the DsRed-expressing bacteria. Further, fluorescence microscopy yielded no information on the membranous compartments surrounding the two pathogens. FIB/SEM tomography is a volume EM technique allowing the acquisition of large-volume tomograms using a focused ion beam/scanning EM, well adapted to the study of 
intracellular pathogens (Kizilyaprak et al., 2019; Weiner and Enninga, 2019). While HIV-1 positive structures engaged in virological synapses with $T$ lymphocytes have been imaged with FIB/SEM (Do et al., 2014; Wang et al., 2017), the compartment had not previously been observed in macrophages with this approach. What was striking from the obtained dataset, is the extensive network of membrane extensions and folds that emanate from the viral compartment as well as from the bacterial vacuole. However, despite these membrane extensions, the compartments were not in direct contact at the time points analysed. Therefore, the invasive bacteria did not seem to take direct advantage of the intracellular niche created by the HIV virus.

However, transient connections between the bacterial- and viral- compartments cannot be excluded. Only one full dataset could be analysed in this study and it is possible that connections could occur at a later time point. In future, potential transient connections could be investigated by loading the viral compartment with a luminal cell tracer before the addition of bacteria labelled with another luminal tracer. Other membranous markers could also be used to visualize the VCC and the SCV (Knuff and Finlay, 2017; Rodrigues et al., 2017).

Considering the volume of membrane, and thus lipids, needed for the production of enveloped viruses in infected cells, hijacking of intracellular trafficking is crucial for the production of new virions. It is likely that the infection of macrophages with HIV-1 leads to profound modifications of intracellular trafficking (Rodrigues et al., 2017). This was observed previously with the sorting protein EHD3 recruited on the viral compartment, and impaired phagolysosome biogenesis and bacterial clearance in HIV-positive macrophages (Dumas et al., 2015). Such changes in intracellular trafficking could therefore benefit any secondary bacterial infection (Le-Bury and Niedergang, 2018). In addition, the major modifications in the gene expression 
programmes of HIV-1 infected macrophages might account for the higher sensitivity of HIV-1-infected cells to subsequent bacterial challenge and defective clearance activity, which is the focus of our current studies. 


\section{Author contribution}

Concept and design of the research, C.D., G.LB. and F.N. ; carrying out the experimental work, A.D., C.D, C.K., G.LB. and F.N. ; providing reagents, J.C.H and M.A.G. Data analysis and interpretation: G.L.B, C.D., J.D., W.B., B.M.H. and C.K. Writing: G.LB., J.C.H and F.N. 


\section{Acknowledgements}

We thank EFS (Saint Vincent de Paul and Trinité) for supply of buffy coats, Dr Anna Mularski for proof reading and Drs O. Steele-Mortimer (NIH USA) and S. Benichou (Institut Cochin) for initial discussions.

\section{Funding}

This work was supported by grants from CNRS, Inserm, Université Paris Descartes,Agence Nationale de Recherches sur le Sida et les Hépatites (ANRS AO2012-2) and Fondation pour la Recherche Médicale (FRM DEQ20130326518) to

F.N. A part of the work was supported by an R'Equip Grant 316030_128692 of the Swiss National Science Foundation (to BMH). C.D. was supported by a post-doctoral grant from ANRS. Both FRM and ANRS contributed to support the PhD salary of G.LB. 


\section{References}

Bakowski, M.A., V. Braun, and J.H. Brumell. 2008. Salmonella-containing vacuoles: directing traffic and nesting to grow. Traffic. 9:2022-2031.

Biggs, B.A., M. Hewish, S. Kent, K. Hayes, and S.M. Crowe. 1995. HIV-1 infection of human macrophages impairs phagocytosis and killing of Toxoplasma gondii. J Immunol. 154:61326139.

Canals, R., D.L. Hammarlof, C. Kroger, S.V. Owen, W.Y. Fong, L. Lacharme-Lora, X. Zhu, N. Wenner, S.E. Carden, J. Honeycutt, D.M. Monack, R.A. Kingsley, P. Brownridge, R.R. Chaudhuri, W.P.M. Rowe, A.V. Predeus, K. Hokamp, M.A. Gordon, and J.C.D. Hinton. 2019. Adding function to the genome of African Salmonella Typhimurium ST313 strain D23580. PLoS Biol. 17:e3000059.

Carden, S., C. Okoro, G. Dougan, and D. Monack. 2015. Non-typhoidal Salmonella Typhimurium ST313 isolates that cause bacteremia in humans stimulate less inflammasome activation than ST19 isolates associated with gastroenteritis. Pathogens and disease. 73.

Collaborators, G.B.D.N.-T.S.I.D. 2019. The global burden of non-typhoidal salmonella invasive disease: a systematic analysis for the Global Burden of Disease Study 2017. Lancet Infect Dis.

Crowe, S.M., N.J. Vardaxis, S.J. Kent, A.L. Maerz, M.J. Hewish, M.S. McGrath, and J. Mills. 1994. HIV infection of monocyte-derived macrophages in vitro reduces phagocytosis of Candida albicans. J Leukoc Biol. 56:318-327.

Do, T., G. Murphy, L.A. Earl, G.Q. Del Prete, G. Grandinetti, G.H. Li, J.D. Estes, P. Rao, C.M. Trubey, J. Thomas, J. Spector, D. Bliss, A. Nath, J.D. Lifson, and S. Subramaniam. 2014. Threedimensional imaging of HIV-1 virological synapses reveals membrane architectures involved in virus transmission. J Virol. 88:10327-10339.

Dumas, A., G. Le-Bury, F. Marie-Anais, F. Herit, J. Mazzolini, T. Guilbert, P. Bourdoncle, D.G. Russell, S. Benichou, A. Zahraoui, and F. Niedergang. 2015. The HIV-1 protein Vpr impairs phagosome maturation by controlling microtubule-dependent trafficking. J Cell Biol. 211:359-372.

Ganor, Y., F. Real, A. Sennepin, C.A. Dutertre, L. Prevedel, L. Xu, D. Tudor, B. Charmeteau, A. CouedelCourteille, S. Marion, A.R. Zenak, J.P. Jourdain, Z. Zhou, A. Schmitt, C. Capron, E.A. Eugenin, R. Cheynier, M. Revol, S. Cristofari, A. Hosmalin, and M. Bomsel. 2019. HIV-1 reservoirs in urethral macrophages of patients under suppressive antiretroviral therapy. Nat Microbiol.

Gilchrist, J.J., and C.A. MacLennan. 2019. Invasive Nontyphoidal Salmonella Disease in Africa. EcoSal Plus. 8.

Gilks, C.F., R.J. Brindle, L.S. Otieno, P.M. Simani, R.S. Newnham, S.M. Bhatt, G.N. Lule, G.B. Okelo, W.M. Watkins, P.G. Waiyaki, and et al. 1990. Life-threatening bacteraemia in HIV-1 seropositive adults admitted to hospital in Nairobi, Kenya. Lancet. 336:545-549.

Gordon, M.A., A.L. Walsh, M. Chaponda, D. Soko, M. Mbvwinji, M.E. Molyneux, and S.B. Gordon. 2001. Bacteraemia and mortality among adult medical admissions in Malawi--predominance of non-typhi salmonellae and Streptococcus pneumoniae. J Infect. 42:44-49.

Herrero-Fresno, A., I. Wallrodt, P. Leekitcharoenphon, J.E. Olsen, F.M. Aarestrup, and R.S. Hendriksen. 2014. The role of the st313-td gene in virulence of Salmonella Typhimurium ST313. PLoS One. 9:e84566.

Holden, D.W. 2002. Trafficking of the Salmonella vacuole in macrophages. Traffic. 3:161-169.

Kedzierska, K., R. Azzam, P. Ellery, J. Mak, A. Jaworowski, and S.M. Crowe. 2003. Defective phagocytosis by human monocyte/macrophages following HIV-1 infection: underlying mechanisms and modulation by adjunctive cytokine therapy. J Clin Virol. 26:247-263.

Kingsley, R.A., C.L. Msefula, N.R. Thomson, S. Kariuki, K.E. Holt, M.A. Gordon, D. Harris, L. Clarke, S. Whitehead, V. Sangal, K. Marsh, M. Achtman, M.E. Molyneux, M. Cormican, J. Parkhill, C.A. MacLennan, R.S. Heyderman, and G. Dougan. 2009. Epidemic multiple drug resistant Salmonella Typhimurium causing invasive disease in sub-Saharan Africa have a distinct genotype. Genome Res. 19:2279-2287. 
Kizilyaprak, C., J. Daraspe, G. Longo, and B.M. Humbel. 2015. Investigation of resins suitable for the preparation of biological sample for 3-D electron microscopy. J. Struct. Biol. 189:135-146.

Kizilyaprak, C., Y.D. Stierhof, and B.M. Humbel. 2019. Volume microscopy in biology: FIB-SEM tomography. Tissue Cell. 57:123-128.

Knuff, K., and B.B. Finlay. 2017. What the SIF Is Happening-The Role of Intracellular SalmonellaInduced Filaments. Front Cell Infect Microbiol. 7:335.

Koppensteiner, H., R. Brack-Werner, and M. Schindler. 2012. Macrophages and their relevance in Human Immunodeficiency Virus Type I infection. Retrovirology. 9:82.

Kroger, C., A. Colgan, S. Srikumar, K. Handler, S.K. Sivasankaran, D.L. Hammarlof, R. Canals, J.E. Grissom, T. Conway, K. Hokamp, and J.C. Hinton. 2013. An infection-relevant transcriptomic compendium for Salmonella enterica Serovar Typhimurium. Cell Host Microbe. 14:683-695.

Lacharme-Lora, L., S.V. Owen, R. Blundell, R. Canals, N. Wenner, B. Perez-Sepulveda, W.Y. Fong, R. Gilroy, P. Wigley, and J.C.D. Hinton. 2019. The use of chicken and insect infection models to assess the virulence of African Salmonella Typhimurium ST313. PLOS neglected tropical diseases. 13:e0007540.

Le-Bury, G., and F. Niedergang. 2018. Defective Phagocytic Properties of HIV-Infected Macrophages: How Might They Be Implicated in the Development of Invasive Salmonella Typhimurium? Frontiers in immunology. 9:531.

Malik-Kale, P., C.E. Jolly, S. Lathrop, S. Winfree, C. Luterbach, and O. Steele-Mortimer. 2011. Salmonella - at home in the host cell. Frontiers in microbiology. 2:125.

Mazzolini, J., F. Herit, J. Bouchet, A. Benmerah, S. Benichou, and F. Niedergang. 2010. Inhibition of phagocytosis in HIV-1-infected macrophages relies on Nef-dependent alteration of focal delivery of recycling compartments. Blood. 115:4226-4236.

Niedergang, F. 2016. Phagocytosis. In Encyclopedia of Cell Biology. Vol. 2. R. Bradshaw and P. Stahl, editors. Waltham, MA: Academic Press. 751-757.

Niedergang, F., and S. Grinstein. 2018. How to build a phagosome: new concepts for an old process. Curr Opin Cell Biol. 50:57-63.

Niedergang, F., J.-C. Sirard, C. Tallichet Blanc, and J.-P. Kraehenbuhl. 2000. Entry and survival of Salmonella typhimurium in dendritic cells and presentation of recombinant antigens do not require macrophage-specific virulence factors. PNAS. 97:14650-14655.

Okoro, C.K., R.A. Kingsley, T.R. Connor, S.R. Harris, C.M. Parry, M.N. Al-Mashhadani, S. Kariuki, C.L. Msefula, M.A. Gordon, E. de Pinna, J. Wain, R.S. Heyderman, S. Obaro, P.L. Alonso, I. Mandomando, C.A. MacLennan, M.D. Tapia, M.M. Levine, S.M. Tennant, J. Parkhill, and G. Dougan. 2012. Intracontinental spread of human invasive Salmonella Typhimurium pathovariants in sub-Saharan Africa. Nature genetics. 44:1215-1221.

Ramachandran, G., D.J. Perkins, P.J. Schmidlein, M.E. Tulapurkar, and S.M. Tennant. 2015. Invasive Salmonella Typhimurium ST313 with Naturally Attenuated Flagellin Elicits Reduced Inflammation and Replicates within Macrophages. PLoS neglected tropical diseases. 9:e3394.

Rodrigues, V., N. Ruffin, M. San-Roman, and P. Benaroch. 2017. Myeloid Cell Interaction with HIV: A Complex Relationship. Frontiers in immunology. 8:1698.

Singletary, L.A., J.E. Karlinsey, S.J. Libby, J.P. Mooney, K.L. Lokken, R.M. Tsolis, M.X. Byndloss, L.A. Hirao, C.A. Gaulke, R.W. Crawford, S. Dandekar, R.A. Kingsley, C.L. Msefula, R.S. Heyderman, and F.C. Fang. 2016. Loss of Multicellular Behavior in Epidemic African Nontyphoidal Salmonella enterica Serovar Typhimurium ST313 Strain D23580. MBio. 7.

Tan, J., and Q.J. Sattentau. 2013. The HIV-1-containing macrophage compartment: a perfect cellular niche? Trends Microbiol.

Torre, D., L. Gennero, F.M. Baccino, F. Speranza, G. Biondi, and A. Pugliese. 2002. Impaired macrophage phagocytosis of apoptotic neutrophils in patients with human immunodeficiency virus type 1 infection. Clin Diagn Lab Immunol. 9:983-986.

Van Puyvelde, S., D. Pickard, K. Vandelannoote, E. Heinz, B. Barbe, T. de Block, S. Clare, E.L. Coomber, K. Harcourt, S. Sridhar, E.A. Lees, N.E. Wheeler, E.J. Klemm, L. Kuijpers, L. Mbuyi Kalonji, M.F. Phoba, D. Falay, D. Ngbonda, O. Lunguya, J. Jacobs, G. Dougan, and S. Deborggraeve. 2019. 
An African Salmonella Typhimurium ST313 sublineage with extensive drug-resistance and signatures of host adaptation. Nat Commun. 10:4280.

Wang, L., E.T. Eng, K. Law, R.E. Gordon, W.J. Rice, and B.K. Chen. 2017. Visualization of HIV T Cell Virological Synapses and Virus-Containing Compartments by Three-Dimensional Correlative Light and Electron Microscopy. J Virol. 91.

Weiner, A., and J. Enninga. 2019. The Pathogen-Host Interface in Three Dimensions: Correlative FIB/SEM Applications. Trends Microbiol. 27:426-439. 


\section{Figure legends}

Figure 1 - Increased intracellular replication and survival of ST313 in both HIV1- and HIV-1+ primary human macrophages, compared with ST19 bacteria (A) Schematic representation of the experimental design. Primary human macrophages were infected with HIV-1 $1_{\mathrm{ADA}} \mathrm{WT}$ or not for 8 days and then co-infected with $S$. Typhimurium ST19 4/74 or ST313 D23580 for 20 min. At this point, designated t=0, cells were washed with media containing gentamicin. At different time points postinfection, cells were lysed or fixed and prepared for plating or imaging. (B) Numbers of intracellular bacteria were assessed by viable count at $20-30 \mathrm{~min}, 4-6 \mathrm{~h}$ or $20-24 \mathrm{~h}$. Each point shows the mean value from three technical replicates for one donor and the points between $\mathrm{HIV}-1^{-}$and $\mathrm{HIV}-1^{+}$macrophages from individual donors are connected. The bar shows the mean value for all donors $(n \geq 3)$ Two-tailed $p$-values by paired $t$-test are shown. (C) Results are expressed as a ratio of the number (CFU) of intracellular ST313, compared with ST19 bacteria. The mean \pm SEM at least of three independent experiments on different donors is presented.

Figure 2 - Localization of S. Typhimurium and HIV-1 following co-infection in primary human macrophages. (A-C) Primary human macrophages were coinfected with HIV-1 $1_{\text {ADAWT }}$ and with S. Typhimurium ST19 4/74 (A, upper panel) or ST313 iNTS D23580 (A, lower panel). Cells were fixed, permeabilized, and labelled with anti-p24 (to localize HIV) followed by AlexaFluor-488-labeled anti-goat IgG (A, green, second column) and anti-Salmonella O-antigen, followed by Cy3-labeled antirabbit IgG (A, red, third column). (A) Maximum Z-projections of $6 \mathrm{~h}$ after infection were represented. Scale bar, $10 \mu \mathrm{m}$. (B) Scatter plot of red (Salmonella-Cy5) and green (HIV-GFP) pixel intensities of cells in (A) infected with ST19 (upper graph) or with 
ST313 (lower graph). (C) Co-localization of HIV p24-positive structures and S. Typhimurium ST313 or ST19 in co-infected cells using the Pearson correlation coefficient (PCC). Each point represents the PCC within one cell, and bars show the mean value for 3 donors. (D-E) Primary human macrophages were co-infected with


between the virus $\left(\mathrm{GFP}^{+}\right)$and the bacteria $\left(\mathrm{DsRed}^{+}\right)$was determined by PCC, using ICY software. Means of 3 cells from 3 independent donors are represented. (E) To visualize S. Typhimurium and HIV, maximum Z-projections (ImageJ) (upper panels), and 3D reconstructions (ICY) (lower panels) were made. Scale bar, $10 \mu \mathrm{m}$.

Figure 3 - Following co-infection, HIV-1 and S. Typhimurium localise to two different compartments in human primary macrophages analysed by FIB-SEM. Primary human macrophages were infected with HIV-1 NLR4.3iGag-GFP for 8 days, and then co-infected with DsRed-expressing ST313 for 6h. (A) Z-stacks of images at 0.3 $\mu \mathrm{m}$ increments were acquired with spinning disk confocal inverted microscope. Maximum Z-projections are represented after image treatment performed using custom-made ImageJ routines. Scale bars, $50 \mu \mathrm{m}$ (left panel) and $20 \mu \mathrm{m}$ (right panel). (B) Representative scanning electron micrograph of a FIB-SEM image stack with a horizontal field of view corresponding to $30 \mu \mathrm{m}$ (i). In this image, bacteria (ii and iii) and HIV vesicles (iv) are identified and manually segmented using IMOD. (C) The final volume is segmented in order to colour the nuclei in blue, the plasma membrane in gold, two different bacteria in red, surrounded by their compartment in purple, and HIV particles in white in the membranous viral compartment (green). (ii and iii) correspond to a zoom in the volume of interest to visualise the compartments in more detail. 


\section{MATERIALS \& METHODS}

Cell culture of human Monocytes-Derived Macrophages (hMDMs) - Blood of healthy donors was used (Etablissement Français du Sang, Ile de France, Site Saint Vincent-de-Paul, Trinité or Saint-Antoine) with the appropriate ethics prior approval as stated in the EFS/ Inserm agreement \#15/EFS/012 and \#18/EFS/030, ensuring that all donors gave a written informed consent, and providing anonymized samples. Primary human Peripheral Blood Mononuclear Cells (PBMCs) were isolated by density gradient sedimentation in Ficoll (GE Healthcare). Then monocytes were selected by adhesion to dishes for $2 \mathrm{~h}$ at $37^{\circ} \mathrm{C}$ in Fetal Calf Serum [FCS]-free medium (RPMI 1640 medium supplemented with 100 Units/mL penicillin and $100 \mathrm{~g} / \mathrm{mL}$ streptomycin (Gibco), and $2 \mathrm{mM} \mathrm{L-glutamine} \mathrm{(Gibco)).} \mathrm{And} \mathrm{they} \mathrm{are}$ differentiated into macrophages for at least 11 days in complete medium (previous medium supplemented with $10 \%$ Fetal Calf Serum) and $10 \mathrm{ng} / \mathrm{mL}$ recombinant human macrophage colony-stimulating factor (rhM-CSF; R\&D systems) (Dumas et al., 2015; Mazzolini et al., 2010).

Viral production and human macrophage infection - Proviral infectious clones of the macrophage-tropic virus isolate ADA (HIV-1 $\left.1_{\mathrm{ADA}} \mathrm{WT}\right)$ have been described previously (Mazzolini et al., 2010). The NLR4.3 HIV-1Gag-iGFP carrying an R5-tropic envelope with the V3-loop V92th014.12 was a kind gift of Michael Schindler (Koppensteiner et al., 2012). Stocks of viral particles were obtained by transfection of HEK293T cells (Human Embryonic Kidney 293, ATCC®CRL-1573 ${ }^{\text {TM }}$, 2x106 ) with 6 $\mu \mathrm{g}$ of the corresponding proviral DNA, using FuGENE® 6 Transfection Reagent as recommended by the manufacturer (Promega). Supernatants of the transfected cells were collected after $48 \mathrm{~h}$, filtered, and stored at $-80^{\circ} \mathrm{C}$. Viral titers were assessed by infection of the indicator cells, HeLa TZM-bl (bearing the $\beta$-galactosidase gene under 
the control of HIV-1 LTR, National Institutes of Health-NIH reagent program), with serial dilutions of the stock, followed by a $\beta$-galactosidase coloration of the cells and counting of blue cells.

At 11 days of macrophage differentiation, HIV-1 virus (MOI 0.2 ) was added to hMDMs. Excess virus was removed after 2 days and cells were kept for 6 days before $S$. Typhimurium infection or functional assays.

Bacterial strains growth conditions and bacterial infection - S. Typhimurium ST313 isolate D23580 and ST19 isolate 4/74 were described previously (Kingsley et al., 2009; Kroger et al., 2013; Okoro et al., 2012). S. Typhimurium SSS18, a chloramphenicol and ampicillin-sensitive derivative of S. Typhimurium ST313 isolate D23580(kind gift from Robert Kingsley, Quadram Institute, Norwich, UK), was used to introduce thethe pMR98-DsRed replicative plasmid expressing DsRed protein by electroporation (Niedergang et al., 2000). Bacteria were grown overnight at $37^{\circ} \mathrm{C}$ under aerobic conditions with shaking in Lysogeny Broth (LB) with $50 \mu \mathrm{g} / \mathrm{mL}$ Ampicillin to maintain selection for pMR98-DsRed. Then, S. Typhimurium was subcultured (starting with dilution of $1 / 30$ ) for $2 \mathrm{~h}$ until late-logarithmic phase. MOI was determined for bacterial suspensions by measuring absorbance at $600 \mathrm{~nm}$, and estimating that $10^{9}$ bacteria/mL give an $A 600$ of 1 . The bacterial suspension was diluted in RPMI 1640 medium supplemented with $2 \mathrm{mM} \mathrm{L-glutamine} \mathrm{to} \mathrm{infect} \mathrm{cells}$ and the inoculum dose was calculated by plating serial dilutions onto LB agar plates.

Gentamicin assays - In 6-well plates, HIV-1 ${ }_{\text {ADA }}$ WT-infected or not infected hMDMs $\left(5.10^{5}\right.$ to $1.10^{6}$ cells/well) were infected at a $\mathrm{MOI}$ of 30 with $1 \mathrm{~mL}$ of bacterial suspension inoculum. Plates were then centrifuged for $2 \mathrm{~min}$ at $500 \mathrm{~g}$ to synchronize the infection and the kinetics was started by incubating the plates at $37^{\circ} \mathrm{C}$ in $5 \% \mathrm{CO}_{2}$. 
After 20 min of incubation at $37^{\circ} \mathrm{C}$, hMDMs were gently washed twice with sterile PBS1X without $\mathrm{CaCl}_{2} / \mathrm{MgCl}_{2}$ (Gibco), and incubated in complete RPMI medium supplemented with $50 \mu \mathrm{g} / \mathrm{mL}$ of gentamic, and this was designated $t=0$. At the indicated times post-infection, cells were washed twice with PBS1X and lysed with $0.5 \%$ Triton $\mathrm{X}-100$ in PBS1X. The number of viable intracellular bacteria present at each time point was determined by plating serial dilutions on LB agar plates.

Imaging fixed cells - In 24-well plates, HIV-1 ${ }_{\text {ADA }}$ WT-infected hMDMs or not $\left(2.10^{5}\right.$ cells/well) were infected at a $\mathrm{MOI}$ of 30 with $0.5 \mathrm{~mL}$ of bacterial suspension inoculum. Plates were then centrifuged for $2 \mathrm{~min}$ at $500 \mathrm{~g}$ to synchronize the infection and the kinetics was started by incubating the plates at $37^{\circ} \mathrm{C}$ in $5 \% \mathrm{CO}_{2}$. After the invasion step at $20 \mathrm{~min}$, hMDMs cells were fixed or were gently washed 2 times with sterile PBS1X (Gibco), and then incubated in complete RPMI medium supplemented with $50 \mu \mathrm{g} / \mathrm{mL}$ of gentamicin for $6 \mathrm{~h}$ before fixation. Cells were fixed with $4 \%$ formaldehyde (PFA - Sigma-Aldrich) with 4\% sucrose for $15 \mathrm{~min}$, then remaining aldehyde groups were inactivated with $\mathrm{NH}_{4} \mathrm{Cl} 50 \mathrm{mM}$ in PBS1X for 7 min at room temperature (RT), and cells were washed with PBS1X. Cells were blocked and permeabilized with $0.05 \%$ saponin, $2 \%$ FCS in PBS1X (PBS/FCS/Sap) before labelling. S. Typhimurium and HIV-1 were labelled with rabbit anti-Salmonella LPS antibodies (Difco) and goat anti-p24 HIV-1 antibodies (AbD Serotec 4999-9007) in PBS/FCS/Sap for 45 min respectively, followed by 30 min for secondary antibodies in PBS/FCS/Sap: donkey Alexa Fluor647-labeled F(ab')2 anti-rabbit IgG and donkey Alexa Fluor 488-labeled F(ab')2 anti-goat IgG (Jackson ImmunoResearch). Nuclei and S. Typhimurium were labelled with 4',6-diamidino-2-phenylindole (DAPI ; Sigma) at $0.3 \mu \mathrm{g} / \mathrm{mL}$ in PBS1X for $5 \mathrm{~min}$. Mounting medium was Fluoromount-G (eBioscience). Image acquisition was performed on an inverted wide-field microscope 
(Leica DMI600) with a HCX PL APO 100X/1.4 numeric-aperture (NA) oil phase contrast (PH) objective (Nikon) and ORCA Flash4.0 (Hamamatsu) camera. Z-series of images were taken at $0.2 \mu \mathrm{m}$ increments and deconvolution was performed with Huygens software (Scientific Volume Imaging) when indicated. Analysis was performed using custom-made ImageJ (National Institutes of Health) routines and colocalization was estimated using JACoP plugin of ImageJ software to calculate the Pearson correlation coefficient (PCC).

Live cell imaging - hMDMs differentiated for 11 days were detached using PBS/EDTA 2 mM, and seeded on gridded MatTeck Glass Bottom Dishes (P35G-214-CGRD) at $1.10^{6}$ cells/well. HIV-1 ${ }_{\text {NLR4.3 Gag-iGFP-infected }}$ hMDMs were infected at a $\mathrm{MOI}$ of 30 with $1 \mathrm{~mL}$ of bacterial suspension inoculum (iNTS SSS18 carrying replicative DsRed plasmid). Plates were then centrifuged for $2 \mathrm{~min}$ at $500 \mathrm{~g}$ to synchronize the infection and the kinetics was started by incubating the plates at $37^{\circ} \mathrm{C}$ in $5 \% \mathrm{CO}_{2}$.

After the invasion step, hMDMs were gently washed twice with sterile PBS1X without $\mathrm{CaCl}_{2} / \mathrm{MgCl}_{2}$, and then incubated in complete RPMI medium supplemented with 50 $\mu \mathrm{g} / \mathrm{mL}$ of gentamicin for $6 \mathrm{~h}$ for acquisition. Samples were observed with a spinning disk confocal (CSU-X1M1; Yokogawa) inverted microscope (DMI6000; Leica) equipped with a CoolSnap $\mathrm{HQ}^{2}$ camera (Photometrics) and a heated chamber at $37^{\circ} \mathrm{C}$ with $5 \% \mathrm{CO}_{2}$ in a BSL3 laboratory. GFP, DsRed and $\mathrm{PH}$ images were acquired every 5 min using HCX PLAN APO 63X/1.4 NA oil PH objective on MetaMorph 7.5.5 software (Molecular Devices). Z-series of images were taken at $0.3 \mu \mathrm{m}$ increments. Image treatments were performed using custom-made ImageJ routines, and 
calculation of Pearson coefficient and 3D reconstructions were performed using ICY (Pasteur Institute) software.

For FIB-SEM analysis, cells were washed with 0.2 M HEPES and then fixed with $2.5 \%$ glutaraldehyde in $0.2 \mathrm{M}$ HEPES for $2 \mathrm{~h}$ at RT.

\section{Focused Ion Beam (FIB)-Scanning Electron Microscope (SEM) tomography -} To identify co-infected cells, HIV-1-GFP and Salmonella-DsRed were observed with a $100 \mathrm{X}, 1.4 \mathrm{NA}, \mathrm{PH}$ DIC objective with a spinning disk confocal (Yokogawa CSUX1M1) inverted microscope (Leica DMI6000) equipped with a CoolSnap $\mathrm{HQ}^{2}$ camera (Photometrics) and a heated chamber with $\mathrm{CO}_{2}$ in a BSL3 laboratory. Acquisition was performed with MetaMorph 7.5.5 (Molecular Devices).

Cells were then post-fixed in a chemical mix containing $2.5 \%$ glutaraldehyde (EMS \# 16300), 1\% osmium tetroxide (EMS \# 19150) and 1.5\% Potassium hexacyanoferrate(II) trihydrate (Sigma \# 60280) in phosphate buffer $(0.1 \mathrm{M}, \mathrm{pH} 7.4)$ (Sigma \# P3619) for $2 \mathrm{~h}$ at RT. Cells were then washed three times for $5 \mathrm{~min}$ in distilled water and dehydrated in graded series of ethanol, (30\% for $5 \mathrm{~min}, 50 \%$ for 5 min, en-bloc stained in 2\% uranyl acetate (EMS \# 22400) in 50\% ethanol for $1 \mathrm{~h}$, $70 \%$ ethanol for $5 \mathrm{~min}, 80 \%$ for $5 \mathrm{~min}, 2$ times $95 \%$ for $10 \mathrm{~min}$, and three times in pure ethanol for $6 \mathrm{~min})$. This was followed by infiltration in epoxy resin $(43.5 \%$ epoxy medium, 36.5\% DDSA, 18\% MNA, 1.5\% DMP30) (Sigma \# 45359) using a drop of fresh epoxy resin on the monolayer overnight and finally polymerized for $48 \mathrm{~h}$ at $60^{\circ} \mathrm{C}$. The epoxy blocks containing the monolayer were glued on aluminium SEM specimen stubs with EPO-TEK H20S conductive resin (EMS \# 12672-20S) as described previously (Kizilyaprak et al., 2015; Kizilyaprak et al., 2019). The stub was mounted in the FIB-SEM (Helios Nanolab 650, FEl Company) using the $45^{\circ}$ aluminium SEM-mount (EMS \# 75230). 
The preparation regime of the block in the FIB-SEM microscope is depicted in Kizilyaprak et al. (Kizilyaprak et al., 2015). Cell of interest (COI) was localized using the gridded reference system on the epoxy block. The FIB-SEM tomography of the $\mathrm{COI}$ was performed with the FEI Slice and View software ${ }^{\mathrm{TM}}$. The stage was tilted to $7^{\circ}$ in order to have the block-surface normal to the ion beam. The milling was done at $30 \mathrm{kV}$ acceleration voltage, $790 \mathrm{pA}$ current and $1 \mu \mathrm{s}$ dwell time using a slice thickness corresponding to $10 \mathrm{~nm}$. For image acquisition the stage was tilted to $45^{\circ}$ to have the freshly milled face normal to the electron beam. The cross-section images were acquired by detecting backscattered electrons with the through-the-lens detector (BSE-TLD) in immersion mode using an electron beam of $2 \mathrm{kV}, 800 \mathrm{pA}$ and $20 \mu$ s of dwell time with a frame of $4096 \times 3536$ corresponding to a pixel size of $7.3 \mathrm{~nm}$. Image stack was aligned by cross-correlation and manually segmented using the IMOD software package (University of Colorado, Boulder, Colorado, USA). A stack with reduced number of sections (1 out of 5 ) is available on line https://imagerie.cochin.inserm.fr/sis4web/home3.php?collection=4740776 for visualization or download. 


\section{A}

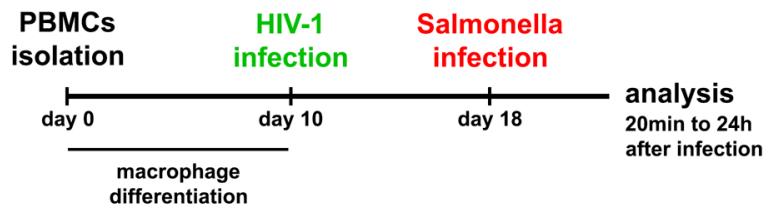

B internalization $\quad 4-6 \mathrm{~h} \quad 20-24 \mathrm{~h}$
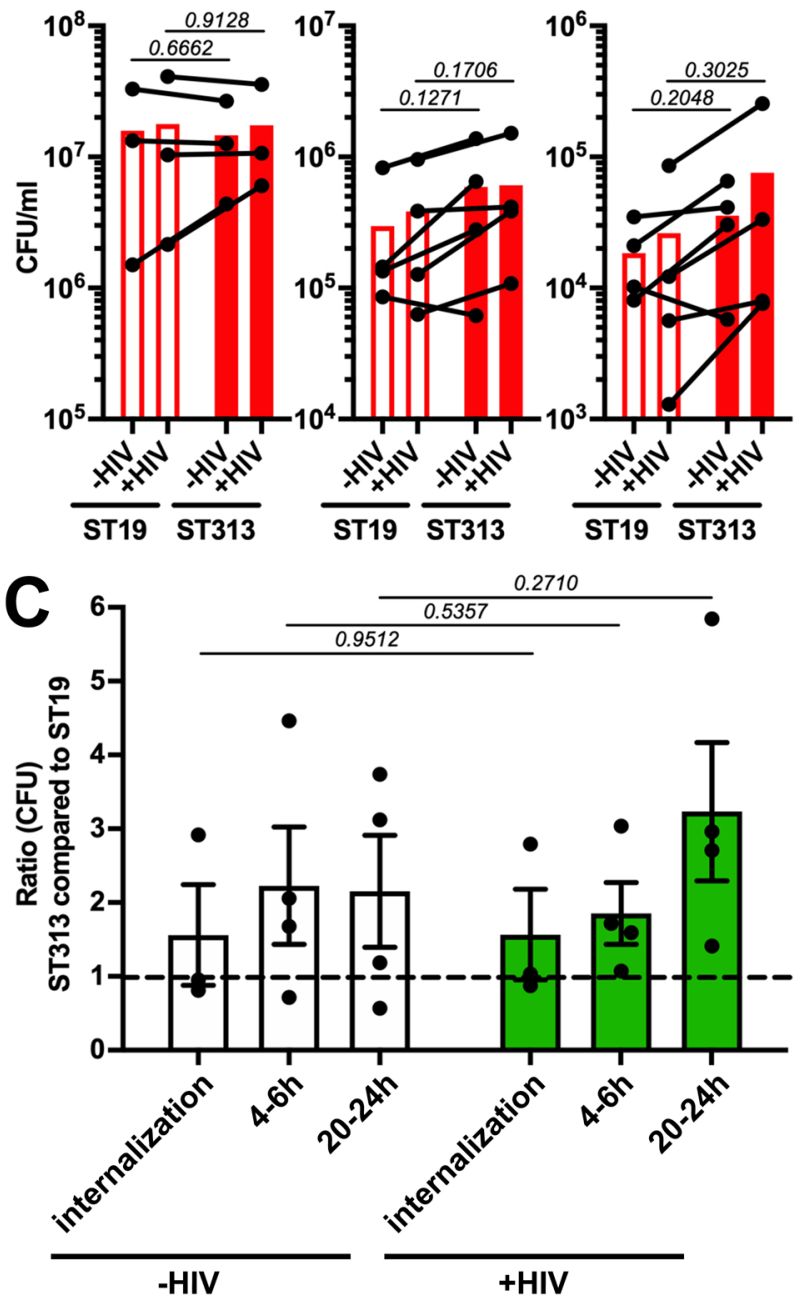

Figure 1 

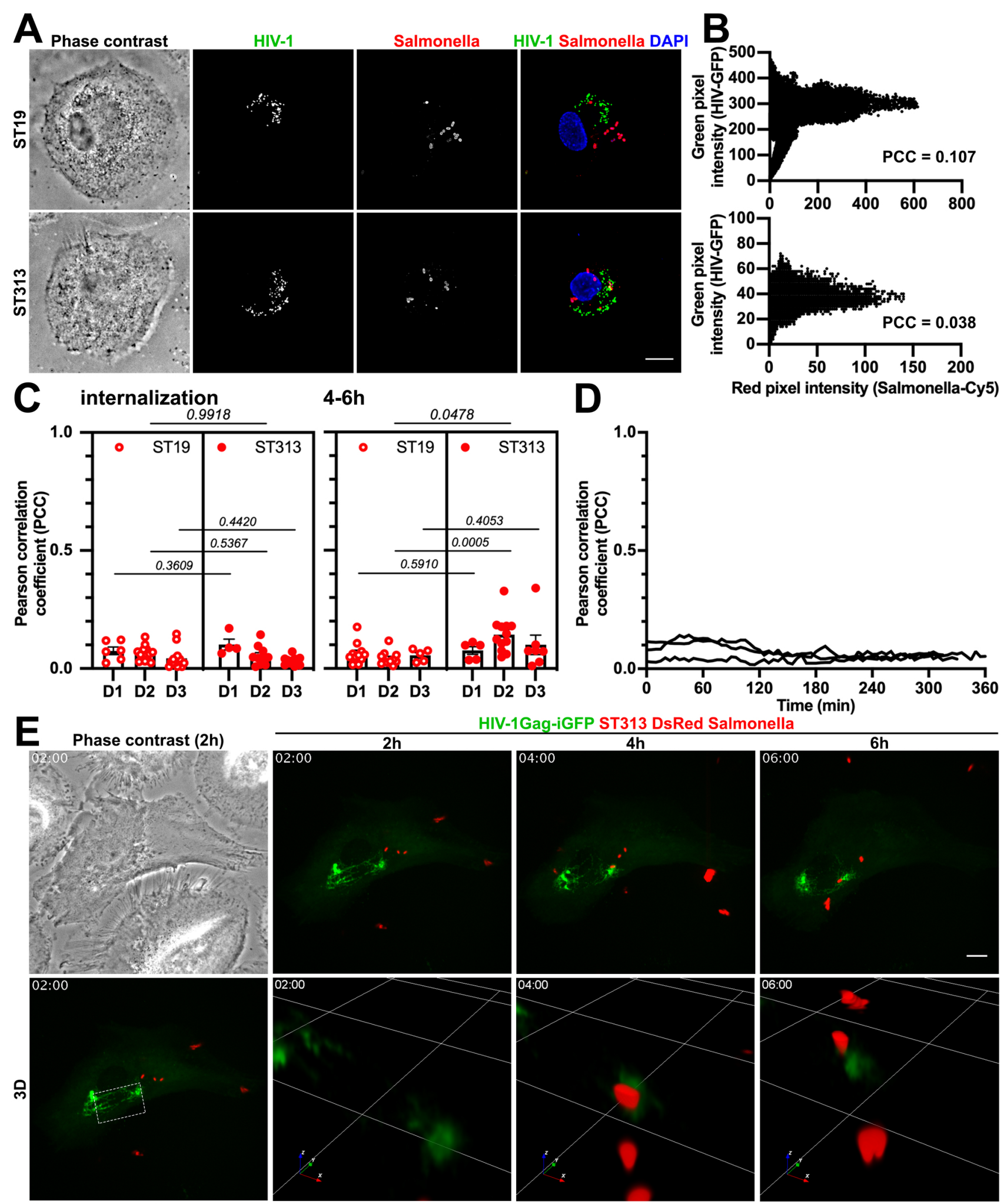

HIV-1Gag-iGFP ST313 DsRed Salmonella
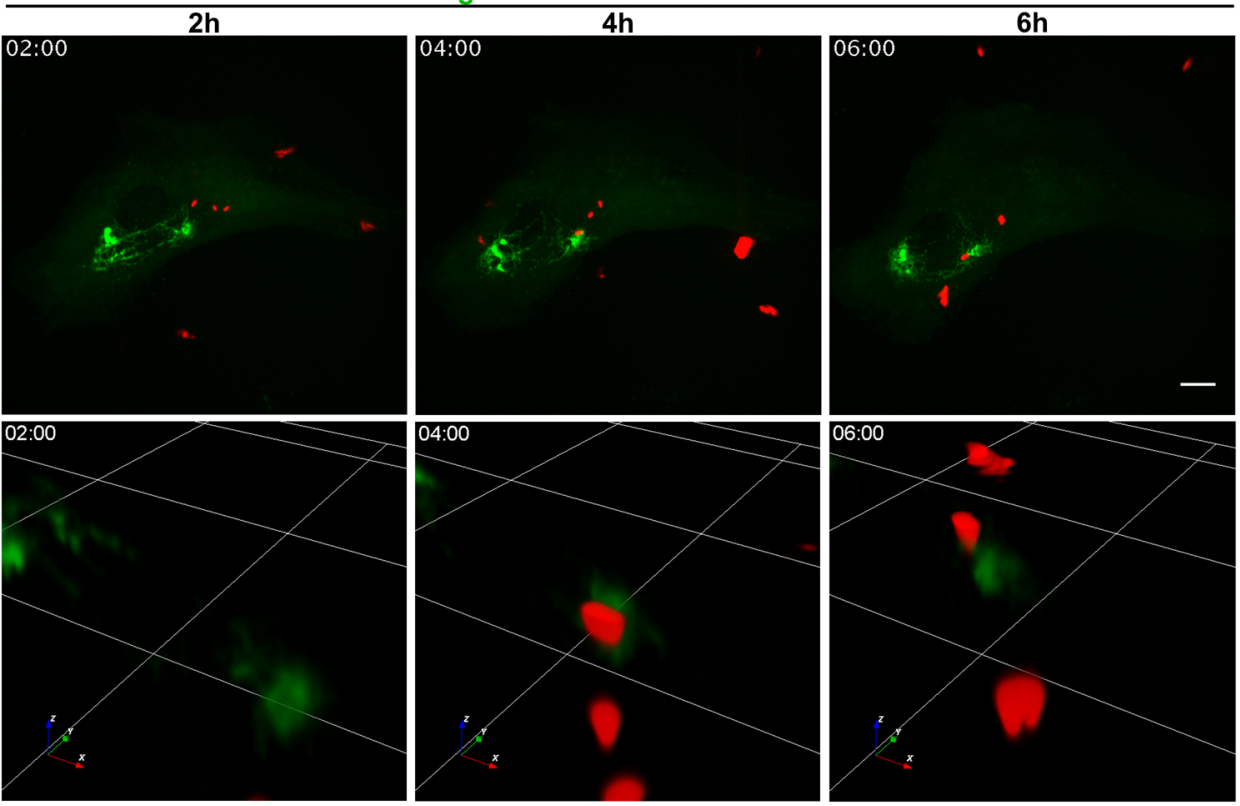

Figure 2 


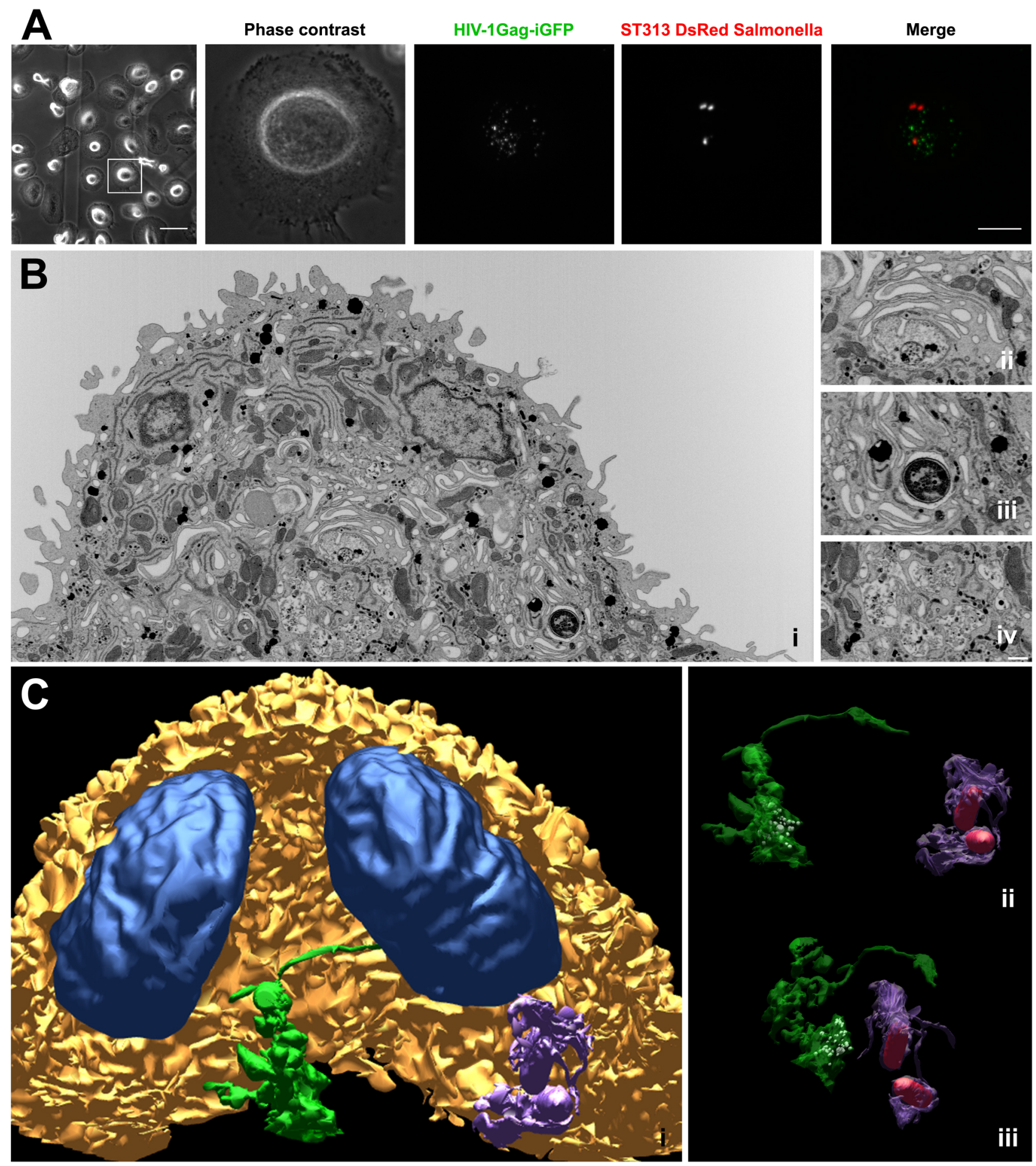

Figure 3 